\title{
Quantifying the mixing due to bars
}

\section{Patricia Sanchez-Blazquez}

\author{
Universidad Autonoma de Madrid, email: psanchezblazquez@gmail.com
}

\begin{abstract}
We will present star formation histories and the stellar and gaseous metallicity gradients in the disk of a sample of 50 face-on spiral galaxies with and without bars observed with the integral field unit spectrograph PMAS. The final aim is to quantify the redistribution of mass and angular momentum in the galactic disks due to bars by comparing both the gas-phase and star-phase metallicity gradients on the disk of barred and non-barred galaxies. Numerical simulations have shown that strong gravitational torque by non-axisymmetric components induce evolutionary processes such as redistribution of mass and angular momentum in the galactic disks (Sellwood \& Binney 2002) and consequent change of chemical abundance profiles. If we hope to understand chemical evolution gradients and their evolution we must understand the secular processes and re-arrangement of material by non-axisymmetric components and viceversa. Furthermore, the re-arrangement of stellar disk material influences the interpretation of various critical observed metrics of Galaxy evolution, including the age-metallicity relation in the solar neighborhood and the local G-dwarf metallicity distribution. Perhaps the most obvious of these aforementioned non-axisymmetric components are bars - at least $2 / 3$ of spiral galaxies host a bar, and possibly all disk galaxies have hosted a bar at some point in their evolution. While observationally it has been found that barred galaxies have shallower gas-phase metallicity gradients than non-barred galaxies, a complementary analysis of the stellar abundance profiles has not yet been undertaken. This is unfortunate because the study of both gas and stars is important in providing a complete picture, as the two components undergo (and suffer from) very different evolutionary processes.
\end{abstract}

\section{Reference}

Sellwood, J. \& Binney, J. 2002, MNRAS, 336, 785 